\title{
Flapless guided implant uncovering (FGIU): Minimally invasive healing abutment connection surgery
}

\author{
Alessandro Lanza ${ }^{1,2 *}$, Fabio Scognamiglio ${ }^{3}$, Gennaro De Marco ${ }^{4}$, Michele Lanza $^{5}$, Felice Femiano ${ }^{5}$ and Gennaro Minervini ${ }^{2,6}$ \\ ${ }^{1}$ Associate professor in Campania University Luigi Vanvitelli, Multidisciplinary department of medical, surgical and dental sciences, via Luigi De Crecchio 7, 80138, \\ Naples, Italy \\ ${ }^{2}$ Chair of dental prosthesis and implantology in Campania University Luigi Vanvitelli, Multidisciplinary Department of Medical, Surgical and Dental Sciences, via \\ Luigi De Crecchio 7, 80138, Naples, Italy \\ ${ }^{3}$ Graduate School of oral surgery, Campania University Luigi Vanvitelli Federico II, 80138, Naples, Italy \\ ${ }^{4}$ Dentist Doctor Campania University Luigi Vanvitelli, Multidisciplinary department of medical, surgical and dental sciences, via Luigi De Crecchio 7, 80138, Naples, Italy \\ ${ }^{5}$ Associate Professor in Campania University Luigi Vanvitelli, Multidisciplinary department of medical, surgical and dental sciences, via Luigi De Crecchio 7, 80138, Naples, Italy \\ ${ }^{6}$ Professor in Campania University Luigi Vanvitelli, Multidisciplinary department of medical, surgical and dental sciences, via Luigi De Crecchio 7, 80138, Naples, Italy
}

\begin{abstract}
Purpose: Endosseous implants can be placed following either two-stage technique requiring second-stage surgery or one-stage technique, which does not involve a second surgical intervention. Although there are few reports in the literature that compare the results of different implant uncovering techniques pain and swelling can be reduced by the use of specific devices in the second stage surgery. The main aim of this manuscript is to present a new minimally invasive technique named "Flapless guided implant uncovering (FGIU)".

Materials and methods: A total number of 120 implants were placed in 20 partially or totally edentulous patients. After osseointegration period the implants in each patients were uncovered in one quadrant with a classic two stage surgery with a mucoperiostal flap and in other quadrant with the FGIU technique. Clinical and radiographic parameters are considered in order to guides the operator through this delicate surgical procedure. The clinical results obtained were compared with those derived with classic flap technique, performed in the same patients at different sites.
\end{abstract}

Results: The FGIU technique has reduced the time of the implant-prosthetic rehabilitation, pain and swelling of the second stage surgery in all patients treated if compared with the classic flap technique.

Conclusion: The minimally invasive technique described can be used with predictability to reduce the treatment times and the discomfort of the patient in the second surgical phase.

\section{Introduction}

The implant treatment represents a valid therapeutic option to replace lost teeth due to various causes with high success and survival rates at medium-long term [1]. This result was achieved thanks to a series of factors including the main ones are:

- Diffusion of protocols and guidelines implants internationally standardized $[2,3]$ $[4,5]$

- The improvement of surgical techniques more safe and predictable

- The development of fixture with ossointegration capability better and faster than ever [6,7]

- The evolution of implant prosthetic components, can now even to individualize the design of the building for each clinical condition $[8,9]$.

In the context of the implant surgical techniques, when you choose to work with the classic two stage technique (implant submerged and covered by soft tissue), you should be careful not only to the positioning of the implant (in a prosthetic guided position) but also to the uncovering technique of the fixture. The uncovering of the implant is a very important moment, because depend on it many aesthetic and functional aspects of the future prosthesis but also the time required to terminate the treatment. In general when you opt for a biphasic implant surgery you must prepare a full-thickness flap in the same area of primary implant surgery, put in evidence the fixture/s, remove the cover screw, select and apply a healing screw adapted to the thickness of the soft tissue and suture the flap (Figure $1 \mathrm{~F}-\mathrm{G}$ ). All this requires an average time of wound healing of about 2-4 weeks before starting the prosthetic phase of rehabilitation (for implant-prosthetic phases see Table 1). Today our implant patients not only ask us more predictable secure and not expensive treatments, but at the same time also faster in relation to the total period of the works. For this reason that we have decided to describe in this work a surgical technique for implant exposure that has the primary objective to reduce the time existing

Correspondence to: Alessandro Lanza, Via Luigi De Crecchio 7, 80138, Naples, Italy, Tel: +39 0815665507, Fax: +39 0815665500, E-mail: alessandro.lanza@unina2.it

Key words: healing abutment connection, second stage surgery, dental implant, mucoperiosteal flap

Received: December 01, 2016; Accepted: December 12, 2016; Published: December 14, 2016 

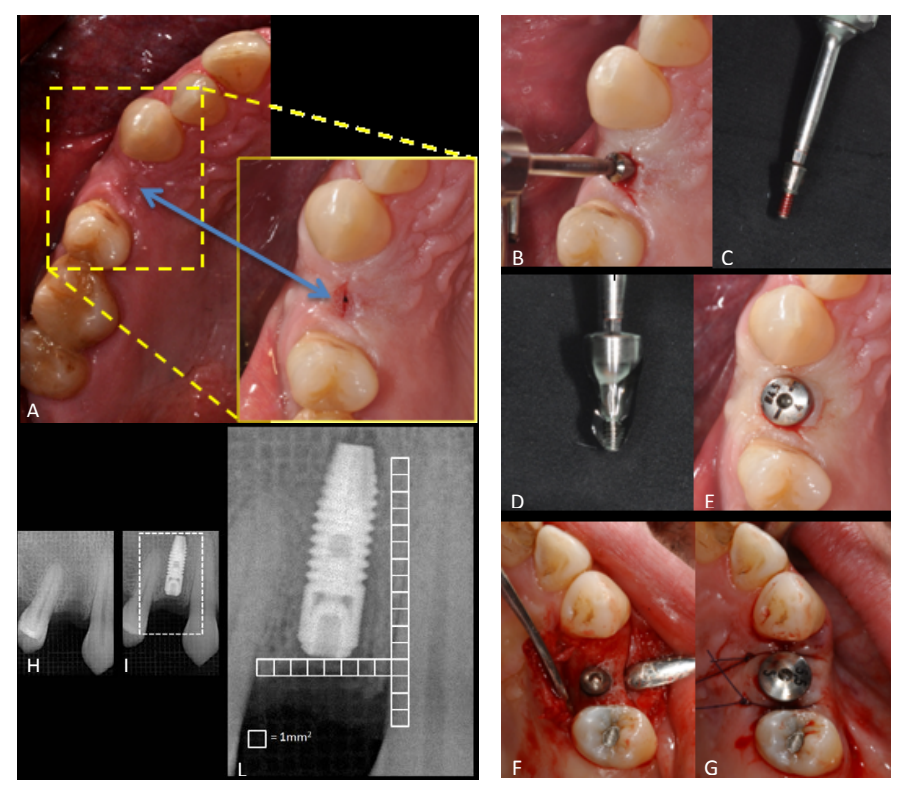

Figure 1. FGIU technique. A: Single submerged implant area (16 weeks healing) and its magnification that shows micro incision uponimplant head. B: Implant cover engaged with hand screw. C: Implant cover removed. D: Healing abutment preparation with ialuronic acid gel. E: Immediate healing abutmentpositioning on implant toward micro incision, note the ischemic compression to around mucosa and the perfect health condition of this, no suture applied. F: Classic flap technique: implant exposed. G: Healing abutment applied and suture done. H: Pre-surgical Endoral Rx. I: implant positioned intraoral Rx. L: I magnification with millimeter scale, useful in pre-exposure making decision.

Table 1. Type of Edentulism and patient's groups: FGIU: Flapless Guided Implant Uncovering Technique; F: Classic Flap Implant Uncovering Technique.

\begin{tabular}{|c|c|c|}
\hline \multirow{2}{*}{} & \multicolumn{2}{|c|}{ Implant Inserted } \\
\cline { 2 - 3 } & FGIU Group & F Group \\
\hline Single Edentulism & 18 & 18 \\
\hline Multiple Edentulism* & 22 & 22 \\
\hline Total Edentulism** & 20 & 20 \\
\hline Total & 60 & 60 \\
\hline
\end{tabular}

$*=2,3$ or 4 implants were positioned in edentulous area

$* *=2,4,6$ implants were positioned in mandible and 4 or 6 implants were positioned in maxilla

Table 2. Differences between FGIU group and F group compared at different times: T0 (day of second stage surgery), T1 (after a week), T2 (1 year follow-up after delivery of the prosthesis)

\begin{tabular}{|c|c|c|c|c|c|c|}
\hline & $\begin{array}{l}\text { FGIU } \\
\text { Group }\end{array}$ & $\begin{array}{c}\text { F } \\
\text { Group }\end{array}$ & $\begin{array}{l}\text { FGIU } \\
\text { Group }\end{array}$ & $\begin{array}{c}\text { F } \\
\text { Group }\end{array}$ & $\begin{array}{l}\text { FGIU } \\
\text { Group }\end{array}$ & $\begin{array}{c}\text { F } \\
\text { Group }\end{array}$ \\
\hline & T0 & T0 & $\mathrm{T} 1$ & T1 & $\mathrm{T} 2$ & $\mathrm{~T} 2$ \\
\hline Mucoperiosteal flap & none & yes & |IIIIIIIIIII & |IIIIIIIII & |IIIIIIIIIII & $\mid / I / I / I / I$ \\
\hline Bledding & none & yes & |IIIIIIIIIII & /IIIIIIIII & |IIIIIIIIIII & /IIIIIIIII \\
\hline Suture & none & yes & |/IIIIIIIIII & |/IIIIIIII & |/IIIIIIIII/ & |/IIII/I/ \\
\hline Contextual preliminary impress & yes & none & none & none & |IIIIIIIIIII & $\mid / I / I / I / I$ \\
\hline Precsion impress & |IIIIIIIIIII & /IIIIIIIIII & yes & none & |IIIIIIIIIII & /IIIIIIIII \\
\hline Post-surgery edema and pain & none & yes & |IIIIIIIIII & /IIIIIIII & |IIIIIIIIII & |IIIIIII/ \\
\hline $\begin{array}{l}\text { Post surgery pharmacologyc } \\
\text { therapy }\end{array}$ & none & yes & |IIIIIIIIIII & /IIIIIIIII & |IIIIIIIIIII & /IIIIIII/ \\
\hline $\begin{array}{l}\text { Perimplant mucosa healing } \\
\text { condition }\end{array}$ & |IIIIIIIIIII & |IIIIIIIIIII & |IIIIIIIIIII & /IIIIIIIII & $\begin{array}{l}\text { very } \\
\text { good }\end{array}$ & $\begin{array}{l}\text { very } \\
\text { good }\end{array}$ \\
\hline
\end{tabular}

between implant surgery and prosthetic phase.

\section{Materials and methods}

A group of 20 patients, female and male ( $12 \mathrm{~F}$ and $8 \mathrm{M})$, with no contraindications to surgery from a systemic point of view (Table 2) were submitted to implant treatment because of lack of at least one dental element in at least two different quadrants. Of the two edentulous sites, one was treated with a classic uncovering technique at second stage surgery with a flap, the other with minimally invasive technique defined FGIU or flapless guided implant uncovering. The results achieved with the two different techniques for each patient were compared at different timing of observation: T0 (same days of implant exposure), T1 (1 week later implant exposure) and T2 (1 year follow-up after cemented prosthesis).

In the 20 patients recruited for the study were uncovered a total of 120 implants of which 18 inserted in singles edentulous sites and treated with a classical flap technique and 18 with FGIU technique, 22 in multiples edentulous sites of 2,3 , or 4 implants treated with classical technique and 22 with technical FGIU, 20 in complete edentulous sites treated with the classic technique (2,4 or 6 to mandibular arch, 4,6 to maxillary arch) and 20 treated with FGIU (Table 2).

The implants used in the study were all Astra Tech dental implant (OsseoSpeed ${ }^{\mathrm{TX}}$ Astra Tech Dentsply Implants, IH S.r.I.). The technique of implant exposure our proposed consists in the exposure of the implant without the preparation of a full-thickness flap but only through a simple incision at the head of the implant. All without sutures and with the possibility to proceed with the first imprint towards the construction of custom impression trays already in the same session of exposure of the implant, reducing not only the invasiveness of surgical reentry but also the overall duration of the treatment. Now we will describe all stages that characterize this technique of implant exposure in order to make it clear and executable by the majority of specialists in this area. It starts with some considerations already during implant placement (it is for this reason that this technique highly recommended for workers involved in the surgical and prosthetic treatment). It would be helpful always to the end of implant surgery run an occlusal photo with fixture placed and an intraoral radiograph applying on the radiographic film a metal millimetric grid to have a clinical and radiographic reference at the second stage surgery (Figure 1). After osseointegration period it is performed anesthetic infiltration only in correspondence of what should be the head implant. From this point of view are very useful photos and endoral rx, before suggested. Given these considerations, you can infiltrate a minimum quantity of anesthetic at the implant heads, using the carpula needle as a probe explorer. After local anesthesia with a micro-blade we perform a linear incision of approximately $2-3 \mathrm{~mm}$ in correspondence of the head implant; with a micro detacher or if possible directly with the manual screwdriver it expose the cover and proceed with the removal of the same (Figure 1). Valued the extent of the transmucosal seal we will choose the most appropriate healing abutment for diameter and height to the specific clinical situation (Figures 2 and 3). The healing abutment is tightened to 10 newtons without application of stitches and at this point you can already make a first impression for the construction of a custom tray. In figure 4 it exposed the surgical sequence. In the twenty patients whose implants were exposed with the technique FGIU, the results at T0 (daily exposure) - T1 (after a week) and T2 (1 year follow-up after delivery of the manufactured) relating to peri-implant soft tissue stability and the overall treatment time were compared with those obtained in the same patients in the sites treated with the classic flap technique (Table 2). The sites treated with the two different technique, were compared also for bleeding, the need for suture, the post-operative symptoms the need of pain and antibiotic therapy, the times to take both the preliminary impression that the precision impression and timing to finish the entire prosthetic rehabilitation. 


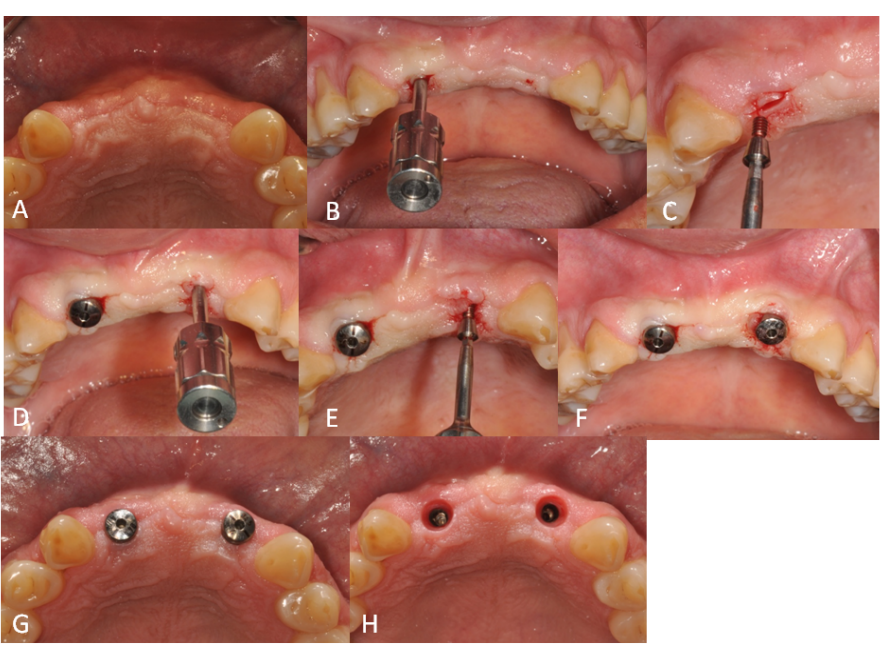

Figure 2. FGIU technique. A: Submerged implant area (16 weeks healing) occlusal view. B: Implant cover engaged with hand screw in site 12. C: Implant cover removed. D: Implant cover engaged in site 22. E: Implant cover removed. F: Immediate healing abutmentpositioning on implant toward micro incision, note the ischemic compression to around mucosa, no suture applied. G: Soft tissue healing around healing abutment 2 week after before taking precision impression $\mathrm{H}$ : Note the perfect healing of the transmucosal seal around the two implants.

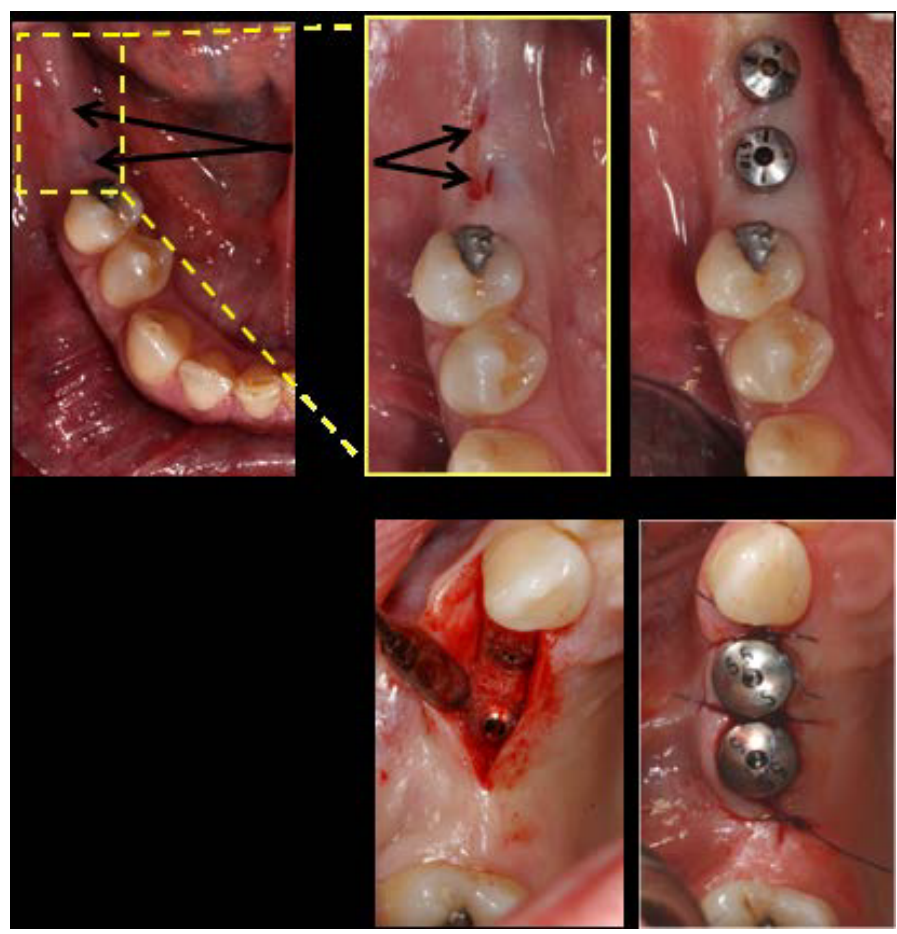

Figure 3. A: Two submerged implant area (16 weeks healing). B: A magnification, micro incision uponimplants head. C: Immediate healing abutmentpositioning on implants toward micro incision, note the ischemic compression to around mucosa and the perfect health condition of this. D: Classic flap technique: implants exposed. E: Healing abutments applied and suture done.

\section{Results}

From the comparative study between patients treated with FGIU technique and classic technique of implant exposure it showed that between the two there are no significant differences in terms of aesthetic and functional results one year after delivery of the prosthesis (T2). However, there are considerable differences at T0 and T1 (supplementary figure). In the specific case, patients treated with
FGIU technique at T0 have not suffered exposure surgery with a flap, consequently there was no bleeding such as to require the suture, it is obtained in most cases a perfect adaptation of peri-implant soft tissue to the healing abutment chosen without dehiscence of the flap. This procedure has allowed taking the preliminary impression for the construction of the custom impression tray already at the time of fixture exposure. Antibiotics were not prescribed. The postoperative course was characterized by little pain only in the first hour's posttreatment controlled with topical application of ice. For all patients at T0 it was taken the preliminary impression in alginate and after one week the subjects in most of the cases were submitted to precision impression with classical technique using the pick-up (except in some cases where exposure was performed in complete edentulous patients). In about the totality of the cases treated with FGIU technique the time between implant exposure and taking the precision impression was just one week.

The implant sites treated with classic exposure technique conversely have undergone flap surgery, more bleeding, application of stitches, had higher discomfort at the post-operative and need for antibiotic therapy associated with pain therapy in most of the cases. In addition considered the largest invasiveness of this treatment and the presence of bleeding compared to sites FGIU, at T0 has not been possible to take the preliminary impression. At T1, the sites treated with classical technique, are checked, you remove the stitches, checking the status of the peri-implant soft tissue inflammation and in most cases postpone the patient to a second control after 7/10 days. Only after perfect peri-implant soft tissue healing (3-4 weeks) was taken the second impression, thus lengthening the treatment times.

\section{Discussion}

The results presented in this study show that the main differences between sites treated with FGIU and those with Classic flap technique occur in the first days of healing from the implant exposure. The main differences noted (TAB2) in T0 and T1 respectively in FGIU patient and classic technique patient, are caused essentially to the minimal invasiveness of the first compared to second technique. A second minimally invasive surgical phase as shown in the figure 5 allows avoiding surgical flaps, to apply the sutures, to use antibiotics and often pain medication. It also accelerates the execution time of the prosthetic work that begins already at T0 with the taking of 'preliminary impression and at $\mathrm{T} 1$ with taking the precision impression
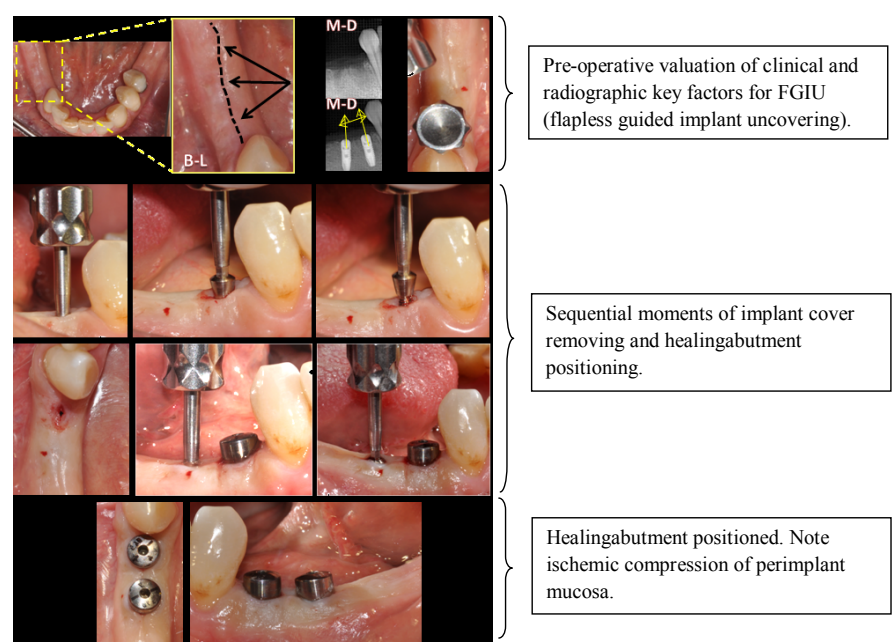

Figure 4. Schematic sequence of FGIU (flapless guided implant uncovering) at T0. 


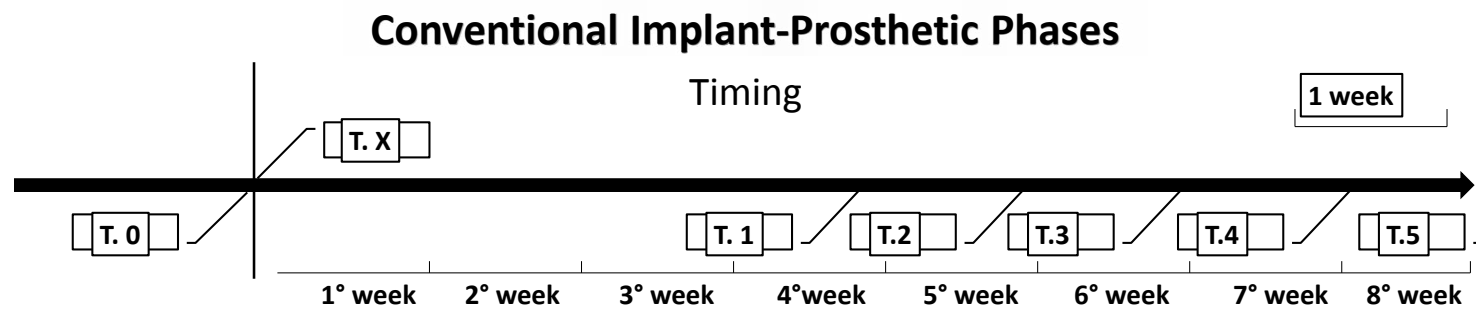

T. 0:Osseointegrated implants $\rightarrow$ implant uncovering (2-4 Months for mandible 4-6 months for maxilla)

T. X:First impression (7 days from T.0)

T. 1:Precision impression (3-4 weeks from second stage surgery/T0 with complete healing of soft tissue )

T. 2:Abutment connection. Clinical and radiographical control of the mesostructure (1-3 weeks from T1)

T. 3:Esthetic and functional control of the prosthetic work (5-6 weeks from second stage surgery)

T. 4:Delivery of the prosthesis (6-8 weeks from second stage surgery)

T. 5:Follow-up

Figure S1. Conventional implant-prosthetic phases.

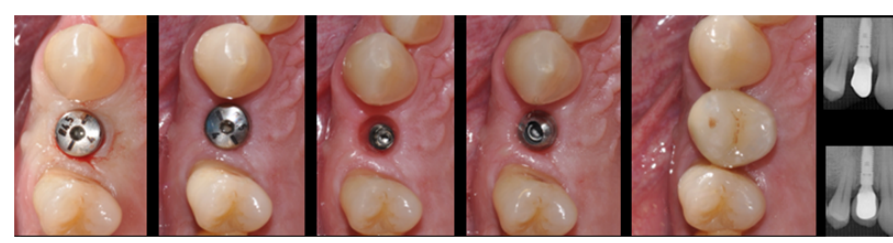

Figure 5. Prosthetic rehabilitation time sequence of implant \#14 (3 weeks total).A: T0= Implant exposure time. B: $\mathrm{T} 1=1$ week later $\mathrm{A}$, precision impress time. $\mathrm{C}$ : $\mathrm{T} 1$, contro of trans-mucosal tissue before precision impress. D: Implant abutment test. E: Fitting radiographic test of second structure on abutment. F: prosthetic crown completed, controlled and cemented. G: Fitting radiographic test of prosthetic crown.

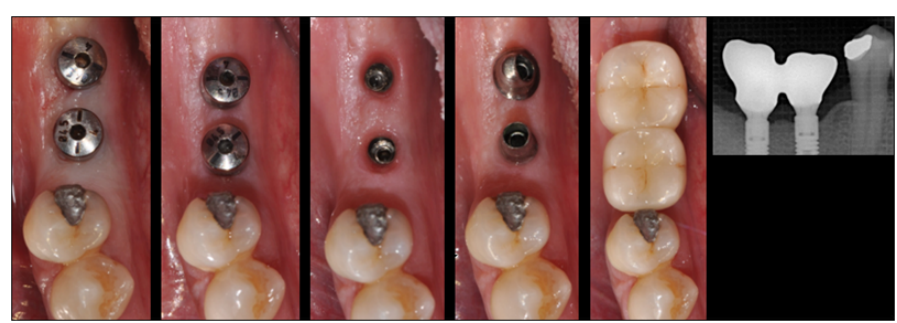

Figure 6. Prosthetic rehabilitation time sequence of implant \#46-47 (3 weeks total).A $\mathrm{T} 0=$ Implant exposure time. $\mathrm{B}: \mathrm{T} 1=1$ week later $\mathrm{A}$, precision impress time. $\mathrm{C}: \mathrm{T} 1$, control of trans-mucosal tissue before precision impress. D: Implant abutment test. E: prosthetic crowns completed, controlled and cemented. F: Fitting radiographic test of second structure on abutments.

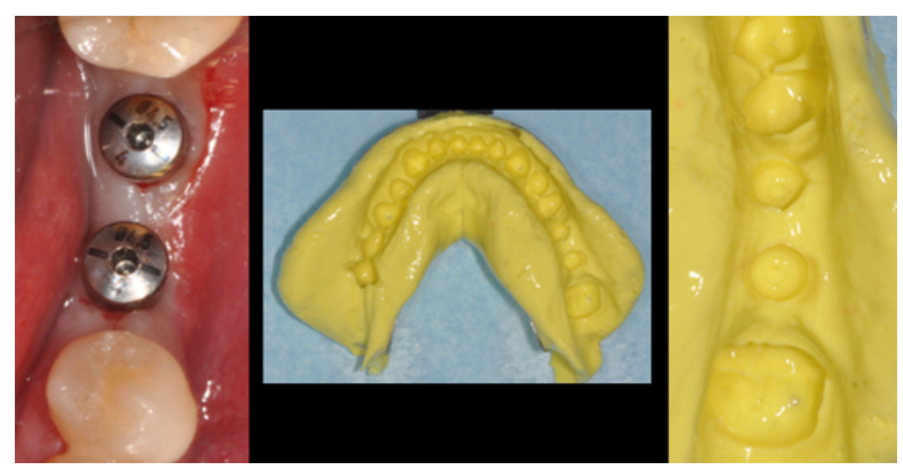

Figure 7. Example of preliminary impression immediately after implant exposure. No flap, no bleeding, no suture, no deishences between abutment and perimplant mucosa are present. and not after 3-4 weeks as normally happens in the sites treated with the classical technique (Figures 6 and 7). In this sense, the FGIU technique meets the functional, aesthetic and temporal requirements of the implant patients, significantly reducing the total treatment times. It is good to consider that these positive results obtained in the sites treated with FGIU, are also the result of the experience of operators who for years are dedicated to clinical research in implantology and who treat each implant patient also from a prosthetic point of view. The limit of this technique is represented by the operator-dependency. In effect, in inexperienced hands technique FGIU may make mistakes not easy to correct than a classic technique. If you are unable to immediately identify the implant head, in effect, the successive and repeated attempts would result in a secure alteration of the quality and quantity of the peri-implant mucosa, conversely very useful for the future survival not only of the fixture but also of the prosthesis from a functional and aesthetic point of view. Given the simple executive protocol adopted by the FGIU, its overall benefits in terms of, minimal invasiveness, comfort and fast execution as well as reducing the time of treatment, we recommend its use in all areas of application of biphasic implant surgery except where you only have a thin gingival biotype or poor keratinized mucosa or when combining primary implantology intervention also with guided bone regeneration that includes the use of non-resorbable barrier membranes. In these cases, the clinician is obliged to set up the flap to see well and completely remove the membrane used. A good surgical and prosthetics skill are essential to better exploit the potential of this technique.

\section{Conclusion}

Today the keywords to success in implantology have become: security, predictability, quality, economy and the short duration of treatment. It is in relation to these aspects of implantology that fits the speech of FGIU technique. It is minimally invasive so safe for the patient, predictable if you follow the recommended protocol, very economic and able to economize from two to four weeks compared to the timing of a standards implant prosthetic treatment. From this point of view, the use of this technique join the monophasic technique to the biphasic technique, in which, waited the time for osseointegration, passes directly to the preliminary impression taking to build the custom impression tray and then continue with the next phases prosthetic. Even in implantology biphasic with FGIU it begins the prosthetic phase on the day of implant exposure with the preliminary impression taking. 
If we explain the benefits of this technique it is worth noting also the limits. First, as already mentioned, the operator must be skilled and preferably also the same that performed the first surgery. It is also not indicated in all areas of implantology. Its application field is the biphasic (two stage) implantology without context guided bone regeneration that involves the use of non-resorbable barrier membranes. In view of what has been shown in this work, we conclude that the evolution in the implantology passes certainly through the use of techniques and principals valid and standardized, in this context, the FGIU technique seems to possess these requirements, then when there is the indication, it would be useful to use it.

\section{References}

1. Wennerberg A, Albrektsson T (2011) Current challenges in successful rehabilitation with oral implants. J Oral Rehabil 38: 286-294.[Crossref]

2. Heitz-Mayfield LJ, Needleman I, Salvi GE, Pjetursson BE (2014)Consensus statements and clinical recommendations for prevention and management of biologic and technical implant complications. Int J Oral Maxillofac Implants 29: 346-350.[Crossref]
3. Gallucci GO, Benic GI, Eckert SE, Papaspyridakos P, Schimmel M, et al. (2014) Consensus statements and clinical recommendations for implant loading protocols. Int J Oral Maxillofac Implants 29: 287-290. [Crossref]

4. Morton D, Chen ST, Martin WC, Levine RA, Buser D (2014) Consensus statements and recommended clinical procedures regarding optimizing esthetic outcomes in implant dentistry.Int J Oral Maxillofac Implants 29: 216-220.[Crossref]

5. Belser U, Buser D, Higginbottom F (2004) Consensus statements and recommended clinical procedures regarding esthetics in implant dentistry.Int J Oral Maxillofac Implants 19: 73-74.[Crossref]

6. Novaes AB Jr, de Souza SL, de Barros RR, Pereira KK, Iezzi G, et al. (2010) Influence of implant surfaces on osseointegration. Braz Dent J 21: 471-481. [Crossref]

7. Le Guéhennec L, Soueidan A, Layrolle P, Amouriq Y (2007) Surface treatments of titanium dental implants for rapid osseointegration. Dent Mater 23:844-854.[Crossref]

8. Wismeijer D, Brägger U, Evans C, Kapos T, Kelly JR, et al. (2014) Consensus statements and recommended clinical procedures regarding restorative materials and techniques for implant dentistry. Int J Oral Maxillofac Implants 29: 137-140.[Crossref]

9. Pabst AM, Walter C, Bell A, Weyhrauch M, Schmidtmann I, et al. (2015) Influence of $\mathrm{CAD} / \mathrm{CAM}$ zirconia for implant-abutment manufacturing on gingival fibroblasts and oral keratinocytes.Clin Oral Investig 19: 1-8. [Crossref]

Copyright: (C2016 Lanza A. This is an open-access article distributed under the terms of the Creative Commons Attribution License, which permits unrestricted use, distribution, and reproduction in any medium, provided the original author and source are credited. 